\title{
At a Glance: An Update on Neuroimaging and Retinal Imaging in Alzheimer's Disease and Related Research
}

\author{
J. Ford', D. Kafetsouli ${ }^{1}$, H. Wilson ${ }^{2}$, C. Udeh-Momoh ${ }^{1}$, M. Politis ${ }^{2}$, S. AhmadiAbhari ${ }^{1}$, I. Rabiner ${ }^{3}$, \\ L.T. Middleton ${ }^{1,4}$
}

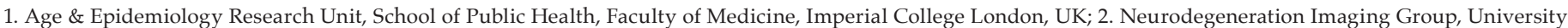

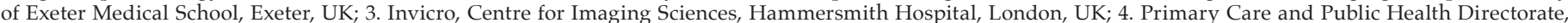
Charing Cross Hospital, Imperial College Healthcare NHS Trust, UK

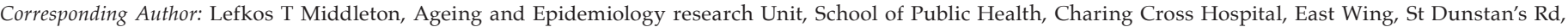
London W6 8RP, United Kingdom, Tel: +44 (0)20 33117290. Email: 1.middleton@imperial.ac.uk

\begin{abstract}
Neuroimaging serves a variety of purposes in Alzheimer's disease (AD) and related dementias (ADRD) research - from measuring microscale neural activity at the subcellular level, to broad topological patterns seen across macroscale-brain networks, and everything in between. In vivo imaging provides insight into the brain's structure, function, and molecular architecture across numerous scales of resolution; allowing examination of the morphological, functional, and pathological changes that occurs in patients across different AD stages (1). $\mathrm{AD}$ is a complex and potentially heterogenous disease, with no proven cure and no single risk factor to isolate and measure, whilst known risk factors do not fully account for the risk of developing this disease (2).

Since the 1990's, technological advancements in neuroimaging have allowed us to visualise the wide organisational structure of the brain (3) and later developments led to capturing information of brain 'functionality', as well as the visualisation and measurement of the aggregation and accumulation of AD-related pathology. Thus, in vivo brain imaging has and will continue to be an instrumental tool in clinical research, mainly in the pre-clinical disease stages, aimed at elucidating the biological complex processes and interactions underpinning the onset and progression of cognitive decline and dementia.

The growing societal burden of AD/ADRD means that there has never been a greater need, nor a better time, to use such powerful and sensitive tools to aid our understanding of this undoubtedly complex disease. It is by consolidating and reflecting on these imaging advancements and developing long-term strategies across different disciplines, that we can move closer to our goal of dementia prevention. This short commentary will outline recent developments in neuroimaging in the field of $\mathrm{AD}$ and dementia by first describing the historical context of $\mathrm{AD}$ classification and the introduction of $\mathrm{AD}$ imaging biomarkers, followed by some examples of significant recent developments in neuroimaging methods and technologies.
\end{abstract}

Key words: Alzheimer's disease, dementia, imaging, biomarkers.

Received October 9, 2021

Accepted for publication December 1, 2021

\section{Introduction}

Tn 1984, the National Institute of Neurological and Communicative Disorders and Stroke (NINCDS) and the Alzheimer's Disease and Related Disorders Association (ADRDA) developed the first set of criteria in the attempts to describe cases of "probable AD" (4). Defined, at the time, as the presence of an acquired amnestic disorder in which at least two cognitive domains (including memory) were measured to be impaired, and this impairment negatively impacted the individual's day-to-day life (4). In 2011, the term "preclinical $\mathrm{AD}^{\circ}$ was introduced as an early stage, along the AD continuum, that acknowledges increased risk among older adults who display no overt clinical symptoms (5-6). The preclinical AD stage is a long phase prior to the onset of measurable mild cognitive decline that warrants a diagnosis of mild cognitive impairment (MCI) due to AD (5). This presents a "long window of opportunity" for targeted secondary prevention measures and interventions (7).

In 2018, the National Institute of Aging / Alzheimer's Association (NIA/AA) expert group proposed the Research Framework biological definition of AD, for research purposes only, based on the in vivo AT[N] triad biomarkers, reflecting the disease pathology of abnormal burden of amyloid (A), tau (T) and neurodegeneration (N) (8). Individuals were classified using a dichotomous construct of positive (+) and negative (-) to symbolise above and below threshold values of these biomarkers (8). This binary classification has subsequently met with criticism pertaining to clinical and practical limitations (9). Asymptomatic (thus cognitively healthy) older adults but still demonstrating biomarkers above a pre-defined threshold - would, under the Research Framework criteria, be categorised as falling along the disease continuum. A concern that was echoed by a recent set of recommendations from the International Working Group (IWG) stating that defining a disease state is not conceivable in the absence of clinical manifestations and that AD-related biomarkers fall along a continuous scale 
and, as such, a binary classification "does not reflect the reality of amyloid- $\beta$ and tau pathology" (10). Our stance on the biological classification of AD to consider carefully the "grey" peri-threshold areas can help to disentangle the complexity of interactions of various risk factors (9).

Non-imaging risk-based assessments measuring various facets of lifestyle (11), genetics (12), and more recently novel blood-based approaches (13) are methods that have proven utility in measuring older adults' increased risk of developing $\mathrm{AD}$ during early, preclinical, disease stages. On-the-other-hand neuroimaging methods are costly and, as yet, not widely available. Nevertheless, the benefits in bringing neuroimaging into the clinical trial space provides invaluable insight into the brains of live human subjects of older adults, across the AD continuum.

The next sections will describe recent developments both in commonly applied neuroimaging modalities, as well as areas that have only until recently been introduced and, still, evaluated. This commentary by no means provides an exhaustive review of all the available methods to explore the brain's structure and function in vivo, but an attempt to provide an update on recent developments and an induction to support the understanding of the many contributions that neuroimaging can offer in $\mathrm{AD} / \mathrm{ADRD}$ research.

\section{Magnetic Resonance Imaging (MRI)}

Structural MRI (sMRI) is a non-invasive imaging modality that can visualise brain anatomy and measure volumetric and other structural pathologies across white and grey matter, by utilising specialised MR sequences. Evidence of loss of volume and neurodegeneration $[\mathrm{N}]$ is one of the triad hallmarks of AD of the NIA/ AA Research Framework and sMRI was acknowledged as a key [N] biomarker tool (14), and those who are suspected to have dementia often receive an MRI scan to ascertain possible signs of neurodegeneration and the extent to which it may be present, as well to exclude unrelated pathologies.

Measuring differences in cortical thickness is one of the several metrices of neurodegeneration based on sMRI. Recent research has highlighted the asymmetric nature of cortical thinning, commonly seen in AD patients (15). Yet, the asymmetrical distribution of cortical thickness is often overlooked in AD research - despite it being an "important feature of normal brain aging that is both shared by and accelerated in neurodegenerative AD" (15). Patients with $\mathrm{AD}$ were found to exhibit steeper reductions in cortical thickness within the left hemisphere, compared to the right, when measured against healthy controls (HC). Suggesting the patterns of neurodegeneration are not symmetrical - particularly in some 'at risk' areas, including the frontal and temporal brain regions. These regions are particularly vulnerable to asymmetrical loss in healthy aging but can exhibit further (accelerated) changes in AD patients (15).
Volumetric measures, calculated from 3D T1-weighted images, have been shown to identify several "brain atrophy subtypes" that can be used to assess neurodegeneration and for AD categorisation (16). MRI scans at baseline have been used to define and differentiate between older adults with (a) "limbic predominant atrophy", (b) "hippocampal sparing atrophy", (c) "typical/diffuse atrophy" and (d) "no evidence of brain atrophy". Those with "typical/diffuse atrophy" and "limbic predominant atrophy" were at increased risk of developing subsequent dementia. These four brain atrophy subtypes were found to be more informative (or sensitive in predicting future dementia incidence) than less specific cortical volume measures - in which sub-categorisation of atrophy patterns could be a potential new biomarker for future studies (16).

The assessment of structural features using MRI also presents valuable opportunities for disease categorisation. For example, cognitive and MRI measures (alongside machine learning [ML] approaches [- covered in more detail below]), were used to aid the classification of participants with $\mathrm{MCI}$, into $\mathrm{AD}$ subcategories (stable [sMCI] and converters to AD [cAD]) (17). The researchers successfully differentiated between sub-groups with the inclusion of MRI features, whereby additions to the models allowed for the measurement of changes in the entorhinal cortex and hippocampal volume. This "mixedeffects derived features" approach in combining cognitive test results and structural MRI was successfully used to measure the long-term trajectories of change within the brain and for the effective prediction and classification of multiple sub-groups. Therefore, the inclusion of imaging biomarkers (in this case MRI) is potentially complimentary with psychometric testing and allows for (a) the detection of early changes in memory function and (b) provided valuable insight into the role of entorhinal cortex as a potential future biomarker in AD imaging studies.

Unlike sMRI, functional MRI (fMRI) measures brain activity. Task-based fMRI paradigms involve exposing participants to stimuli or asking them to engage in cognitive tasks and measuring the elicited neural responses. Alternatively, 'task-free' paradigms collect resting-state fMRI data with the participant simply lying in the MRI scanner without such engagement with stimuli or tasks. For both task and task-free paradigms, blood oxygen level dependent response (BOLD) contrasts are used to measure the changes in blood oxygenation and serving as a proxy for neural activity.

Resting state fMRI has recently been used to investigate potential "compensatory mechanisms" among patients with "MCI due to $\mathrm{AD}^{\prime}$ and $\mathrm{HC}$ (18). Using connectivity metrics such as degree centrality - i.e., the extent to which a node (e.g., a brain region) is connected to all other nodes within a network, the researchers found evidence of "compensatory" regions of interest (ROI). These included the right superior parietal gyrus, the 
right- and the left precentral gyri, and the right middle frontal gyrus. In these ROI, an increased degree centrality was observed, suggesting "more robust connectivity, despite regional atrophy". Increased degree centrality among some ROI was associated with increased cognitive performance, despite localised aggregation of amyloid and neurodegeneration. These findings suggest that neural atrophy and brain functional decline are not necessarily co-dependent as previously thought, and that resting state functional connectivity measures may be useful for studies on neural compensation (18).

Both task-based and task-free fMRI data can measure functional connectivity (assessing for similarities in BOLD signal fluctuations between brain voxels or ROI), and recent research has attempted to use functional connectivity approaches to predict AD-related pathology (19). The authors adopted connectome-based predictive modelling (CPM) to predict CSF p-tau/A 342 (termed the "PATH-fc" model), derived from measures of functional connectivity in those with MCI and AD at a macroscale spatial resolution. Whilst successfully predicting pathology, this approach also predicted rates of cognitive decline and captured alterations in well-cited resting state networks (e.g., the default mode network, among others). Thus, a whole-brain model, derived from measures of alterations in functional connectivity, can successfully help predict two major outcomes of risk factors of $\mathrm{AD}$ (pathology and cognitive decline).

Increasingly the field is becoming aware of the significant contributions that vascular-related risk factors have on AD/ADRD development (20). fMRI data can be utilised to investigate changes in vascularity and is emerging as a potential new biomarker to measure $\mathrm{AD}$ risk. 4D flow MRI can quantify blood velocities across the whole brain, and to measure changes in cardiac pulse pressure and speed (21). This is particularly useful for measuring arterial stiffness and overall "vascular compliance" (21). Arterial spin labelling-MRI (ASLMRI) uses the hydrogen atoms present in water as a proxy for blood flow (21). Advanced imaging protocols used in mouse models e.g., multi-time echo ASL-MRI, can identify microscopic changes in blood-brain barrier permeability using a dynamic contrast-enhanced MRI when comparing age-related water permeability changes (22).

Vascular markers can be visualised using a multitude of imaging modalities. Lacunes, white matter hyperintensities (WMH), and microbleeds are visible using T2*-weighted imaging, susceptibility weighted images, and fluid-attenuated inversion recovery (FLAIR; (23)). FLAIR can measure evidence of cerebrovascular pathology and image processing techniques in this domain has continued to evolve. Recently, when testing the diagnostic accuracy of multiple image segmentation algorithms, estimating WMH when excluding more diffuse, smaller "lower-intensity" volumes led to a higher correlation between WMH and eventual AD clinical diagnosis and reduced cognitive performance, in the presence of abnormal amyloid burden (24).

Furthermore, improvements in imaging protocols e.g., fast BOLD scans to estimate variability in cardiorespiratory frequencies among AD patients (25). These fast-imaging sequences allow for the detection of frequency oscillations attributed to cardiac, respiratory, and other physiological based sources. As an example, variances in frequency oscillations were observed in $\mathrm{AD}$ patients (25). The authors hypothesised that these may be driven by cerebral hypoperfusion, alongside evidence of small vessel disease pathology (e.g., cerebral amyloid angiopathy) that may provide a novel measure in this population, in addition to small vessel reactivity and blood flow velocity in individual penetrating arteries (25).

Whilst the main emphasis of imaging studies in $\mathrm{AD} / \mathrm{ADRD}$ research focuses on grey matter, diffusion weighted imaging (DWI) is the only non-invasive, albeit highly informative neuroimaging modality, that can provide valuable information pertaining to the microstructural connectivity of white matter (WM) tracts of the brain. Achieved by measuring the movement (diffusion) of water molecules within tissues (26). The introduction of DWI followed from a seminal paper on the topic in 1994 that proved the anisotropic diffusion of water can be exploited to understand WM fibre tract orientation (27). Now, there is increasing understanding in how alterations in diffusion signal between AD stages suggestive of changes in the microstructural integrity of WM tracts (26).

However, DWI's main limitation resides in its low specificity, as "cross-networks" of fibres are complex, overlapping structures that cannot be detected using conventional DWI methods. An exciting development in diffusion MRI is the development of fixel-based analysis (FBA). Like a voxel as a volume element (3D), a fixel is a fibre element, and therefore FBA has the potential to expand on the field of tractography extensively, allowing to measure axon density, intra- and extracellular water, and evidence of demyelination and inflammation. Recently a two-year longitudinal study was conducted using participants from the Alzheimer's disease neuroimaging initiative (ADNI) study (28). The authors identified specific fascicles associated with AD development and utilised two primary measures of freewater fraction (FW; a neuroinflammation proxy) and "apparent fibre density" (AFD). Significant increases in FW were found, within multiple ROIs, to be associated with disease's progression in AD patients but not among $\mathrm{MCI}$ and $\mathrm{HC}$ individuals. On the other hand, AFD was found to decrease within multiple ROI in both $\mathrm{AD}$ and MCI patients (by 7-8 and 3-5\% respectively)- suggesting a shared feature between these stages. These new findings exemplify the potential utility of FBA in exploring changes in white matter microstructural integrity between AD stages (28).

Thus, MRI is a powerful tool in that it can measure changes in both grey and white matter depending on its calibration. Moreover, multimodal MR approaches 
combining imaging modalities aimed at exploring changes in both grey and white matter may aid in unravelling some of the pathogenic mechanisms underlying AD development.

The dysregulation of neural iron content is emerging as another risk-factor associated with $\mathrm{AD}$ development that can be visualised using MRI. An imbalance in iron homeostasis is implicated in the development of AD proteinopathies, e.g., $A \beta$ plaques and neurofibrillary tangle (NFT) accumulation (29). Several methods have been shown to measure iron dysregulation, such as "quantitative susceptibility mapping" (QSM). QSM is a non-invasive technique that can be used as a proxy for iron content (30). QSM signal in the inferior temporal gyrus (ITG) has been associated with the degree of cognitive impairment, with the ITG showing to be preferentially affected by tau deposits, suggesting a relationship between iron dysregulation and tau accumulation (30). Importantly, not just iron dysregulation has been implicated in $\mathrm{AD}$ but also copper, aluminium, zinc (and other metal ions) that interact with amyloid and tau in complex ways (31).

\section{Positron Emission Tomography (PET)}

MRI has demonstrated its flexibility as an in vivo and non-invasive imaging modality in AD/ADRD research - crucial for assessing for evidence of structural atrophy (neurodegeneration; [N] within the AT[N] framework), as well as changes in brain function. Unlike MRI, Positron emission tomography (PET) provides a noninvasive means to visualise and measure the density of biologically relevant molecular targets at subnanomolar concentrations, such as amyloid (A) and tau (T). In tandem, both MRI and PET are instrumental in vivo biomarker tools, in AD/ADRD research trials, reflecting AD pathology. During PET studies, radioactive ligands are injected into the bloodstream and bind to the molecular target in question, and their kinetics at the target can be quantified via the emitted gamma radiation captured using the PET scanner. PET uses various metrics to quantify the specific molecular target within ROI e.g., the standard uptake value ratio (SUV) - which is regional concentration of ligand, controlling for the individual's weight and the dose injected (32). A refinement of this approach designed to partially address potential differences in peripheral kinetics of the radioligand, is the standardized uptake value ratio (SUVR), where the SUV in the target region is normalised to a reference region (33). While most PET ligands commonly used in $\mathrm{AD}$ research do not have a true reference region, the use of a pseudo-reference region, which is known to remain relatively stable, has provided significant improvements over the SUV.

Quantification of misfolded proteins such as amyloid and tau is particularly challenging in the context of typical multi-site studies conducted by a large number of imaging centres which require appropriate control of site/scanner qualification/set-up, acquisition, and harmonising analysis. Such studies demand robust analysis processes and pipelines to ensure adequate signal-to-noise and sufficient statistical power for PET studies that may be relatively expensive to conduct. The centiloid scale has recently been introduced, and widely adopted for both amyloid and tau radioligands, that has proven incredibly useful in standardising and harmonizing measurements between research studies and across different radioligands (34). Centiloid values are calculated by linearly scaling the tracer measurement to give a value ranging from 0 to 100 (30). A value of " 0 " refers to the average uptake of the measured tracer comparable to a "young control", and on the other end of the scale, a value of "100" is representative of the average uptake found among "typical AD patients at the dementia stage" (34). Another significant advance has been the development of the AmyloidIQ and TauIQ methods that provide a generalised metric such as "amyloid load" (A $\beta \mathrm{L})$ and have been demonstrated to provide significantly greater power than standard analytical methods (35-37).

PET is a widely used imaging modality in AD/ ADRD research, that has proven its flexibility in targeting specific molecules. Two well-known types are fluorodeoxyglucose- (FDG) and amyloid-PET that have become ubiquitous research tools in the field and the subsequent focus of hundreds (if not thousands) of publications over the years. One of the first radio-ligands, FDG-PET has a wide range of clinical applications in multiple disease areas, as it allows the measurement of regional alterations in glucose metabolism (both hyper- and hypo-metabolism). On-the-other-hand, amyloid-PET provides quantitative data on the regional amyloid-beta $(\mathrm{A} \beta$ ) plaque burden (38). Several amyloid tracers have recently been developed, with significantly increased half-lives, allowing for their industrial (vs site radiochemistry) production; indeed, three such radioligands are currently, widely available commercially: 18F-Florbetapir, 18F-Florbetaben, and 18F-Flutemetamol (38). There is no doubt that both FDG and amyloid-PET have vastly increased our knowledge and understanding of AD progression (and has been widely reported as such). As to not directly echo previous articles on the topic, this article will be instead focusing on tau-PET, a comparatively more novel approach. Tau-PET holds incredible promise in broadening our understanding of AD / ADRD even further, whilst also complimenting FDGand amyloid-PET in multimodal imaging studies.

Tau-PET involves a tau-labelled radiotracer, allowing the visualisation of cerebral tau load. The existing tau ligands provide a good signal of $3 \mathrm{R}$ tau isoform, that features in $A D$, but have poor affinity for the $4 R$ isoform that is more commonly present in tauopathies, such as progressive supranuclear palsy (PSP), and in frontotemporal dementia (FTD). The spread of 
tau pathology was until recently believed to cascade throughout the brain in a predictable or stereotypical pattern outlined by the Braak's tau staging system. However, this has been questioned recently, with four distinct tau trajectory phenotypes proposed (39). Using from the first-generation tau ligand 18F-flortaucipir, the following spatiotemporal subtypes were proposed (a) limbic, (b) medial temporal load (MTL)-sparing, (c) posterior, and (d) lateral temporal. These features may broaden our understanding in explaining interindividual differences among patients across the AD continuum. The "limbic" pattern subtype was found to occur later and was the most frequently found among patients, demonstrating characteristics typically associated with AD (e.g., amnestic symptoms) and a greater proportion were Apolipoprotein $\varepsilon 4$ (APOE ع4) carriers. By comparison, the patients with the "MTLsparing" phenotype had a younger onset, the "posterior" pattern was associated with comparatively slower cognitive decline, and the "lateral temporal" subtype was associated with a more rapid clinical progression in multiple cognitive domains (39).

It has also recently been reported that the global signal intensity of tau-PET (but not $\mathrm{A} \beta-\mathrm{PET}$ ) predicted the level and spatial distribution of cortical atrophy over a one-year period (36). There appeared to be a sequential relationship between aggregated tau and so-called "downstream" neural degeneration leading the transition from MCI to AD (40). A clustering-based approach was used to identify three AD atrophy subtypes (a) "hippocampal-sparing (frontoparietal predominant)", (b) "limbic-predominant (medial temporal lobe predominant)" and (c) "typical (temporal predominant)". Using the $18 \mathrm{~F}-\mathrm{Flortaucipir}$ radiotracer, in MCI and AD patients, the tau burden was greatest among those displaying the hippocampal-sparing and "typical" atrophy subtypes, with the former patients showing the most rapid cognitive decline (one-year post-PET), and the latter, the most pronounced WMH volumes of the atrophy subtypes. Whereas, among those with "limbicpredominant" atrophy, tau burden was especially present within the entorhinal cortex. The sub-classification of atrophic subtypes may, thus, allow to disentangle disease heterogeneity, improve diagnosis capabilities, and support future clinical development (41).

The above outlined the utility of tau-PET to inform our understanding of the distribution of cortical atrophy, but tau-PET can also make valuable contributions alongside brain function in multimodal imaging studies. A notable recent example was the combinational use of resting state fMRI and tau PET data (42). Higher rates of tau accumulation were associated with increased resting-state functional connectivity and brain regions with highertau accumulation rates were preferentially connected to other regions that showed high tau accumulation (as with lower tau accumulation and low tau areas) suggesting a spatial affinity for its propagation between functionally connected regions (42).

As tau-PET is becoming increasingly recognised as a valuable tool, new "second generation" radiotracers are being developed, with higher affinity for tau aggregates, such as 18F-MK-6240, 18F-PI2620, and 18F-APN1607. These radiotracers were shown to also address the liability of first-generation ligands for offsite binding (presumed to be MAO-B). 18F-MK-6240 was found to accurately discriminate between 'A $\beta$ negative' without cognitive impairment and those on the "AD continuum", defined here as 'A $\beta$ positive' with or without cognitive impairment. These results are promising in 18F-MK6240 being utilised to broaden our understanding of AD progression. The authors concluded that longitudinal studies with larger sample sizes would be needed to address the large variance in the results of their study (43).

Another recently developed tau radiotracer that has promising applications in AD research is 18F-PI-2620. It has shown a high binding affinity to aggregated tau in the brain that bolstered a high signal-to-noise ratio in both $\mathrm{AD}$ and $\mathrm{HC}$ participants with good tolerance (44).

Ascertaining the precise aetio-pathogenic mechanisms of AD / ADRD presents an undoubtedly complex problem but imaging protocols, using multiple tracers may be used during a study protocol to better understand the nature of the spatial aggregation of AD pathology and it's sequencing along numerous clinical pathways. Recent evidence suggests that, when measuring tau in the cerebrospinal fluid (CSF) and amyloid- and tau-PET, CSF measuring phosphorylated tau (p-CSF) and CSF totaltau started to increase before the threshold for amyloid PET "positivity" (45), whereas other studies suggest that tau-PET start to progress after amyloid PET positivity. The effects of amyloid PET on tau-PET may be mediated by phosphorylated tau species, with high p-CSF levels predicting increases in tau-PET uptake levels (45). This expands on Braak's staging of neurofibrillary pathology in that tauopathy - cytoskeletal alterations within neurons - (a) follows a "predictable sequencing pattern" that tends to stem from the transentorhinal region (and the basal temporal neocortex), and (b) these intraneuronal changes (e.g., NFTs) precede the aggregation of insoluble amyloid deposits (29). The aggregation and accumulation of NFTs and $A \beta$ are not mutually exclusive events but the understanding of the pathological temporal sequencing of AD across multiple stages is undoubtedly important for developing new treatments and interventions for AD.

As the field is increasingly recognising the role of vascular risk factors in AD development, multimodal imaging studies have begun to explore the synergistic relationships between AD pathological markers and vascular risk. In a study involving preclinical older adults, a significant interaction was found between higher measures of vascular risk and higher levels of $\mathrm{A} \beta$ burden in most regions (bar the entorhinal cortex) that was then associated with increased tau levels (46). 
These results were gathered using a combination of the "Framingham Heart Study cardiovascular disease risk" to measure "vascular risk" (measures of body mass index, history of diabetes, smoking behaviours, among others), 18F-Flortaucipir (tau), and 11C-Pittsburgh Compound-B $(\mathrm{PiB})$ PET $(\mathrm{A} \beta)$, that raises the interesting possibility of increased vascular risk inducing a "second hit" - "that further potentiates the spread of $A \beta$-related neocortical tau pathology" (46). Elevated vascular risk may, thus, present an important avenue in future $\mathrm{AD}$ intervention studies and/or to attenuate the impact of AD-related pathology in older adults.

It may be tempting to focus research and development efforts primarily on tau, given the history of unsuccessful drug development trials focusing on anti-amyloid targeting medication - that is, until the recent FDA "accelerated" approval of aducanumab (47). However, several recent studies emphasise the significant role that $\mathrm{A} \beta$ seems to play in the temporal sequencing of events leading to a clinical diagnosis of AD. A recent study on the histopathological interactions between global amyloid and medial temporal neuroinflammation (48) reported that, in relation to tau spread, amyloid appeared to interact with transentorhinal neuroinflammation which, in turn, triggered the spread of tau across the neocortex (Braak stages II-III), followed by a subsequent spread of tau across Braak stages III-IV and the associated brain regions. A sentiment that was supported a year later, among a group of researchers who concluded that a "moderate" A $\beta$ level is required for tau within the neocortex to be detectable, and that $>40$ centiloid of global $\mathrm{A} \beta$ burden is required to induce an accelerated spread of tau (49).

Another potential PET target is neuroinflammation. Targeted PET radiotracers can be used to visualise the presence of activated microglia that respond to inflammation and injury, within the central nervous system (50). A recent immunofluorescence study, using the AD PDAPPJ20 mice model, found evidence of "autophagic impairment"; as the mice aged, the hippocampus showed lower phagocytic activity when exposed to $A \beta$, indicative of an impairment to effectively promote $A \beta$ clearance from the brain. "Impaired autophagy and lysosome dysfunction" in AD mice may provide a novel research avenue for future studies (51).

The initial radiotracers aimed at visualizing microglial activation and neuroinflammation targeted the translocator protein $18 \mathrm{kDa}$ (TSPO). The first TSPO tracer was 11C-PK11195 followed by 11C-PBR28, 18F-DPA-714 and several others.

In attempting to gain insight into the relationship between neuroinflammation and AT[N] biomarkers, a recent longitudinal study used 11C-PK11195, 11C-PiB PET (amyloid) and 18F-Flortaucipir (tau) in MCI patients, over a two years' period (52). The authors reported that among MCI patients displaying with low $\mathrm{A} \beta$ burden at baseline, but subsequently rising levels of such, showed higher microglial activation that were then found to decline as the individuals approached $\mathrm{A} \beta$ loads at "AD levels". As tau tangles form later along the temporal pathological sequencing in $A \beta+$ individuals, increased tau aggregation was then associated with higher levels of neuroinflammation. The "two peak hypothesis" was therefore proposed, stipulating that the first peak is driven by $A \beta$ aggregation, and the second peak being driven by the accumulation of tau tangles (52).

Non-TSPO neuroinflammation radiotracers have also been developed. For example, the PET tracer 11CBU99008, targeting imidazoline 2 binding sites (I2-BS; found mainly in the mitochondria) was used recently to explore astrocyte reactivity among MCI due to AD or AD patients, compared to healthy controls (53). The authors found a relationship between 11C-BU99008 and 18F-Florbetaben uptake (in support of previous research finding a link between increased astrocytic activity and above threshold $A \beta$ deposition). A further key finding was the observed increased 11C-BU99008 uptake among a significant portion of those classified as "A $\beta$-negative", suggesting that astrocyte reactivity may still occur at below-threshold $\mathrm{A} \beta$ burden. These findings may add credence to the potential value of neuroinflammation as a target for therapeutic intervention.

The development and application of other novel radioligands allow for the exploration of other potential mechanistic pathways that can lead to AD development. A specific example are novel PET tracers that attempt to elucidate the complex relationships between the dysregulation of mitochondria in older adults in connection with alterations in energy metabolism and oxidative stress. Recently, two PET tracers have been developed for in vivo human studies of mitochondrial function: 18F-BCPP-EF and 11C-SA4503 (54). The mitochondrial complex-1 (MC-1) is the first enzyme in the electron transport chain (ETC) critical for the generation of mitochondrial adenosine triphosphate (ATP), and a major source of reactive oxygen species in the cell. [18F]BCPP-EF binding reflects electron transport chain (ETC)-related mitochondrial function. The $\sigma-1$ receptor $(\sigma 1 \mathrm{R})$ is a chaperone protein enhancing $\mathrm{Ca} 2+$ influx from the endoplasmic reticulum into the mitochondria, thus modulating mitochondrial (ATP) production (54). These tracers demonstrated high reliability in the quantification measurements of mitochondrial function in the brains of healthy human participants and have been applied to $\mathrm{AD}$ (and other neurodegenerative diseases) research over the last few years.

A notable example is with a recent study aimed to assess for mitochondrial and glycolytic impairments in patients among those with early to moderate AD (55). The core aim was to explore whether there are regional differences of glycolysis (the breakdown of glucose and measured via [18F]FDG) and of mitochondrial oxidative activity ([18F]BCPP-EF) among AD patients. The authors found reductions in $[18 \mathrm{~F}] \mathrm{BCPP}-\mathrm{EF}$ binding within 
various regions of the temporal (both medial and lateral) cortex; the parahippocampus appeared particularly susceptible to alterations in [18F]BCPP-EF availability, whereas the same region was least affected by [11C] $\mathrm{PiB}$. Thus, in the context of the temporal sequencing of $\mathrm{AD}$, parahippocampal mitochondrial dysfunction may precede hypometabolism (at least in this brain region).

Finally, to further illustrate the flexibility of PET as an in vivo tool for $A D$ and dementia research, the [11C] UCB-J radiotracer has been developed to target synaptic vesicle glycoprotein 2 (SV2; a presynaptic membrane glycoprotein expressed in almost all synapses) that can be used to measure the distribution of synaptic density across the brain (56). [11C]UCB-J was used to identify evidence of synaptic pathology among older adults with a diagnosis of amnestic MCI (aMCI; (56)). The research was conducted in response to prior results finding localised reductions in SV2A binding within the hippocampus among those with aMCI and mild AD $(56,57)$, found an inverse correlation between global $\mathrm{A} \beta$ deposition ([11C] $\mathrm{PiB})$ and hippocampal synaptic density ([11C]UCB-J) among those with aMCI but not with dementia. The authors concluded that this may be explained by $\mathrm{A} \beta$ reaching a "relative plateau as a pool of primarily insoluble fibrillar $A \beta$, a point at which $A \beta$ may uncouple from neurodegenerative processes including synaptic loss". However, it is worth emphasising that 11C radiotracers (e.g., those exemplified in this commentary) are often limited by their incredibly short half-lives, and therefore, require an on-site cyclotron which is not feasible for most clinical research centres.

\section{Retinal Imaging (RI)}

The neurosensory retina, described as 'a window to the brain', given its direct connection to the central nervous system via the retinal ganglion cells (RGC) and ease-of-access, is emerging as a potentially valuable medium for studying AD in vivo (58). Given the eyebrain link, it is not surprising that the retina manifests similar pathological attributes that are evident in the neurodegenerative process. Indeed, AD patients present with retinal degenerative abnormalities such as RGC preferential loss, thinning of the optic nerve and vascular changes have been found in the retina of patients with $\mathrm{MCI}$ and $\mathrm{AD}$ (59). Increasing evidence also confirmed the presence of canonical $\mathrm{AD}$ pathologies ( $\mathrm{A} \beta$ and tau) within the retina and optic nerve $(60,61)$.

Posited as low-cost and non-invasive, retinal imaging technologies are emerging as potentially useful AD biomarker tools in AD research. One such example is Optical Coherence Tomography (OCT), an imaging technique widely used in ophthalmology to generate high-resolution 2D/3D images of retinal anatomy over a wide field of view. Notably, recent OCT studies in MCI and $\mathrm{AD}$ patients have shown evidence of retinal fibre layer (RNFL) thinning, RGC loss and micro-vascular abnormalities $(62,63)$. Furthermore, in a prospective longitudinal setting, lower RNFL thickness at baseline was associated with increased risk of subsequent cognitive decline and dementia $(64,65)$. However, lack of a standardization in OCT studies is a major limitation in its wider use as a clinical research tool (66).

Confocal scanning laser ophthalmoscopy (cSLO), allowing for retinal fluorescence scanning, represents another promising new RI technology in AD research, as it has been shown to directly visualize amyloid deposits in the retina of MCI and mild to moderate AD patients, previously given oral Curcumin (67). Indeed, the food additive curcumin has natural fluorescent properties and binds to fibrillary $A \beta$ thus enabling the identification of plaques with non-invasive retinal fluorescent imaging. Recently, Ngolab et al. (68) used this technique to compare the retinal (curcumin binded) with brain A $\beta$ burden, as detected by PET, in a small sample of asymptomatic participants above and below amyloid threshold; they observed a distinct discriminatory capacity for distinguishing cognitively healthy older adults with high cerebral amyloid load from those with below-threshold amyloid load. Recent evidence suggests that amyloid retinal accumulation may be detected without the use of Curcumin or another reagent, through blue-light autofluorescence scanning (69). This new technique is currently being adopted in our on-going, industry funded, CHARIOT PRO study at Imperial College London (70) and the recently initiated UK-wide "Deep and Frequent Phenotyping (DFP)" study. Assuming that the above preliminary findings are further validated in these and other large-scale prospective longitudinal studies, retinal fluorescence scanning, and OCT may become important low-cost and non-invasive $\mathrm{RI}$ techniques allowing for risk assessment-for-AD in cognitively healthy individuals and in pre-selecting potential RCT participants for the far more expensive and more invasive PET and/or CSF studies for determining $\mathrm{AT}[\mathrm{N}]$ status.

\section{Expanding role of Machine Learning and Artificial Intelligence in Imaging analysis}

Given the mammoth amount of information collected during the acquisition process, imaging is fundamentally a "big data" problem. Analysing such high-dimensional data collected from multiple participants' brain slices, often over several sessions, and even against the data of other imaging methods may be daunting but by being cognisant of the tools available to handle such a challenge is key; and one such approach is via ML.

Amongst many ML techniques, a recent study combining convolutional neural networks $(\mathrm{CNN})$ and ensemble learning (EL) found $\mathrm{CNN}$ as an effective tool in automated feature learning with the use of a variety of multilayer perceptrons", whilst EL effectively integrates multiple models (71). The authors adopted a CNN- 
EL method alongside structural MRI data to classify participants between (a) those with $\mathrm{AD}$ vs. HC, (b) MCIconverters and $\mathrm{HC}$, and (c) MCI-converters vs. MCInonconverters. This data-driven approach proved more accurate in distinguishing the $\mathrm{AD}, \mathrm{MCI}$-converters, and $\mathrm{HC}$ control groups (but not MCI-converters vs. MCInonconverters) compared to other similar methodologies.

Furthermore, 'deep learning' provides an effective means to analyse data collected from multiple sources, in this case imaging (MR), genetic (single nucleotide polymorphisms), and clinical testing data with the aim to support AD staging analysis (72). Deep learning algorithms are advantageous over "shallow" methods due to their ability to learn which features are most predictive for a particular outcome (in this case AD staging using multimodal data). In short, the researchers found that integrating data from multiple sources improved the predictive accuracy between different $\mathrm{AD}$ stages (cognitively normal, MCI, and AD). Supporting deep learning models in future $\mathrm{AD}$ trials to improve the predictive accuracy in staging categorisation (72).

\section{Future Directions}

Despite years of exhaustive research and thousands of publications, we have still not fully elucidated the biological mechanisms and processes underpinning the development of $\mathrm{AD}$ and ADRDs, nor the extent and mechanisms underlying its heterogeneity. A paradigm shift going beyond the exclusive examination of AT[N] biomarkers is warranted to reflect on the strategic longterm considerations in how best to use neuroimaging in $\mathrm{AD} / \mathrm{ADRD}$ research in the future to support our goals in dementia prevention, and as such we suggest a threepronged approach:

1. Consolidating the wealth of new information that neuroimaging studies continue to give us and by using emerging imaging methods to promote the discovery of new biomarkers that may provide important insight into $\mathrm{AD}^{\prime}$ s underlying causes and reasons behind its heterogeneity within studies with long follow-ups and appropriate sample sizes.

2. It is becoming increasingly clear that multimodal neuroimaging studies, possibly coupled with genetic and other fluid biomarkers studies, may be necessary to untangle the effects of multiple risk factors on $\mathrm{AD}$ development. Novel imaging protocols may prove instrumental in uncovering the underlying neural mechanisms behind AD that cannot be achieved using singular imaging methods alone. With emerging neuroimaging and other multi-modal biomarker discovery and validation work, novel analytic ML/artificial intelligence (and other cutting-edge methodologies) are urgently required and are, indeed, beginning to be applied to AD/ADRD research.

In dementia prevention, whereas MRI and PET methodologies remain instrumental tools in randomised clinical trial (RCT) protocols, in pre-clinical $\mathrm{AD}$ stages, their use for risk status assessment in the context of preventative public health strategies in the general population is neither feasible nor clinically relevant. Further research is warranted towards the development and validation of low-cost and noninvasive technologies, such as retinal imaging, plasma biomarkers and genetics, that are amenable to a wider implementation. Such technologies may also be of value in the pre-screening phase of RCTs for selection of candidates for the more expensive and/or invasive neuroimaging or CSF studies.

3. Neuroimaging is by no means a standardised practice at this point in time (73). Given the sheer magnitude of decisions made at various stages, whether it is calibrating the technology or analysing the vast quantities of data, these decisions can significantly affect the outcomes and results. Consequently, often poor reproducibility between research teams remains an ongoing issue. It is only by coming together to determine 'best practice' procedures in how imaging data is collected, recorded, and analysed, can then be disseminated with confidence.

In conclusion, whereas singular neuroimaging techniques may effectively address hypothesis specific objectives, the wealth of options and emerging broad capabilities of neuroimaging makes it invaluable in clinical research to enhance our understanding of this complex and potentially highly heterogeneous disease. The choice is dependent upon the desired outcomes of the clinical research, and the right "tool" for the job depends on the targeted hypothesis and explicit outcome measure(s). The fast pace of the field of neuroimaging in new modalities, more effective data acquisition protocols, and integrative approaches means that brain imaging will continue to play an important part in AD/ADRD research. By reflecting on recent developments in this field, we can make a unified effort to consolidate and branch into new research avenues in the attempts to disentangle the complexity of this relentlessly progressive and devastating disease.

Conflict of Interest: Prof. Lefkos Middleton has received research funding (to Institution) from Janssen, Merck (USA), Gates, Takeda/ Millenium, Novartis, EIT Health, and Invincro, outside the submitted work. Prof M Politis has received grants from Michael J Fox Foundation, City Electrical Factors, Roche Pharmaceuticals, Life Molecular Imaging, Invicro, personal fees from Roche Pharmaceuticals and from Movement Disorder Society, outside the submitted work. Dr E A Rabiner is a full time employee of Invicro, a company conducting commercial studies to support industry and academic research.

Open Access: This article is distributed under the terms of the Creative Commons Attribution 4.0 International License (http:/ / creativecommons.org/ licenses/by/4.0/), which permits use, duplication, adaptation, distribution and reproduction in any medium or format, as long as you give appropriate credit to the original author(s) and the source, provide a link to the Creative Commons license and indicate if changes were made.

\section{References}

1. Leuzy A, Heurling K, Ashton NJ, Schöll M, Zimmer ER. In vivo Detection of Alzheimer's Disease. Yale J Biol Med. 2018 Sep;91(3):291-300. 
2. Rajasekhar K, Govindaraju T. Current progress, challenges and future prospects of diagnostic and therapeutic interventions in Alzheimer's disease. RSC Adv. 2018;8(42):23780-804.

3. Raichle ME. A brief history of human brain mapping. Trends in Neurosciences. 2009 Feb;32(2):118-26.

4. McKhann G, Drachman D, Folstein M, Katzman R, Price D, Stadlan EM Clinical diagnosis of Alzheimer's disease: Report of the NINCDS-ADRDA Work Group* under the auspices of Department of Health and Human Services Task Force on Alzheimer's Disease. Neurology. 1984 Jul 1;34(7):939 939.

5. Sperling RA, Aisen PS, Beckett LA, Bennett DA, Craft S, Fagan AM, et al. Toward defining the preclinical stages of Alzheimer's disease: Recommendations from the National Institute on Aging-Alzheimer's Association workgroups on diagnostic guidelines for Alzheimer's disease. Alzheimer's \& Dementia. 2011 May;7(3):280-92.

6. McKhann GM, Knopman DS, Chertkow H, Hyman BT, Jack CR, Kawas CH, et al. The diagnosis of dementia due to Alzheimer's disease: Recommendations from the National Institute on Aging-Alzheimer's Association workgroups on diagnostic guidelines for Alzheimer's disease. Alzheimer's \& Dementia. 2011 May;7(3):263-9.

7. Isaacson, R. and Saif, N., 2020. A missed opportunity for dementia prevention? current challenges for early detection and modern-day solutions. The Journal of Prevention of Alzheimer's Disease, pp.1-3.

8. Jack CR, Bennett DA, Blennow K, Carrillo MC, Dunn B, Haeberlein SB, et al. NIA-AA Research Framework: Toward a biological definition of Alzheimer's disease. Alzheimer's \& Dementia. 2018, Apr;14(4):535-62.

9. McRae-McKee K, Udeh-Momoh CT, Price G, Bajaj S, de Jager CA, Scott D, et al. Perspective: Clinical relevance of the dichotomous classification of Alzheimer's disease biomarkers: Should there be a "gray zone"? Alzheimer's \& Dementia. 2019 Oct;15(10):1348-56.

10. Dubois B, Villain N, Frisoni GB, Rabinovici GD, Sabbagh M, Cappa S, et al. Clinical diagnosis of Alzheimer's disease: recommendations of the International Working Group. The Lancet Neurology. 2021 Jun;20(6):484-96.

11. Zhang, X., Tian, Y., Wang, Z., Ma, Y., Tan, L. and Yu, J., 2021. The Epidemiology of Alzheimer's Disease Modifiable Risk Factors and Prevention. The Journal of Prevention of Alzheimer's Disease, pp.1-9.

12. Daunt, P., Ballard, C., Creese, B., Davidson, G., Hardy, J., Oshota, O., Pither R. and Gibson, A., 2020. Polygenic Risk Scoring is an Effective Approach to Predict Those Individuals Most Likely to Decline Cognitively Due to Alzheimer's Disease. The Journal of Prevention of Alzheimer's Disease, pp.1-

13. Keshavan, A., 2020. Population-based blood screening for preclinical Alzheimer's disease in a British birth cohort at age 70. Brain, 144(2), pp.434449.

14. Jack CR, Bennett DA, Blennow K, Carrillo MC, Feldman HH, Frisoni GB, et al. A/T/N: An unbiased descriptive classification scheme for Alzheimer disease biomarkers. Neurology. 2016 Aug 2;87(5):539-47.

15. The Australian Imaging Biomarkers and Lifestyle Flagship Study of Ageing, Roe JM, Vidal-Piñeiro D, Sørensen $\varnothing$, Brandmaier AM, Düzel S, et al. Asymmetric thinning of the cerebral cortex across the adult lifespan is accelerated in Alzheimer's disease. Nat Commun. 2021 Dec;12(1):721.

16. Planche V, Bouteloup V, Mangin J, Dubois B, Habert M, Tison F, et al. Alzheimer's disease brain atrophy subtypes are associated with incident dementia in subjective cognitive complaint and mild cognitive impairment: Neuroimaging / Optimal neuroimaging measures for early detection. Alzheimer's \&amp; Dementia [Internet]. 2020 Dec [cited 2021 May 30];16(S5). Available from: https: / onlinelibrary.wiley.com/doi/10.1002/alz.039861

17. Mofrad S, Lundervold A, Vik A, Lundervold A. Cognitive and MRI trajectories for prediction of Alzheimer's disease. Scientific Reports. 2021;11(1).

18. Behfar Q, Behfar SK, von Reutern B, Richter N, Dronse J, Fassbender R, et al. Graph Theory Analysis Reveals Resting-State Compensatory Mechanisms in Healthy Aging and Prodromal Alzheimer's Disease. Front Aging Neurosci. 2020 Oct 22;12:576627.

19. Prakash R, McKenna M, Gbadeyan O, Shankar A, Andridge R, Scharre D. A Whole-Brain Functional Connectivity Model of Alzheimer's Disease Pathology. 2021;

20. Cheng Y, Chiu M, Chen Y, Cheng T, Lai Y, Chen T. The contribution of vascular risk factors in neurodegenerative disorders: from mild cognitive impairment to Alzheimer's disease. Alzheimer's Research \& Therapy. 2020;12(1).

21. Rivera-Rivera LA, Cody KA, Eisenmenger L, Cary P, Rowley HA, Carlsson $\mathrm{CM}$, et al. Assessment of vascular stiffness in the internal carotid artery proximal to the carotid canal in Alzheimer's disease using pulse wave velocity from low rank reconstructed 4D flow MRI. J Cereb Blood Flow Metab. 2021 Feb;41(2):298-311.

22. Ohene Y, Harrison IF, Evans PG, Thomas DL, Lythgoe MF, Wells JA. Increased blood-brain barrier permeability to water in the aging brain detected using noninvasive multi-TE ASL MRI. Magn Reson Med. 2021 Jan;85(1):326-33.

23. Badji A, Westman E. Cerebrovascular pathology in Alzheimer's disease: Hopes and gaps. Psychiatry Research: Neuroimaging. 2020 Dec;306:111184.
24. Tubi MA, Feingold FW, Kothapalli D, Hare ET, King KS, Thompson PM, et al. White matter hyperintensities and their relationship to cognition: Effects of segmentation algorithm. NeuroImage. 2020 Feb;206:116327.

25. Tuovinen T, Kananen J, Rajna Z, Lieslehto J, Korhonen V, Rytty R, et al. The variability of functional MRI brain signal increases in Alzheimer's disease at cardiorespiratory frequencies. Sci Rep. 2020 Dec;10(1):21559.

26. Vogt NM, Hunt JF, Adluru N, Dean DC, Johnson SC, Asthana S, et al. Cortical Microstructural Alterations in Mild Cognitive Impairment and Alzheimer's Disease Dementia. Cereb Cortex. 2020 May 14;30(5):2948-60.

27. Basser P, Mattiello J, LeBihan D. MR diffusion tensor spectroscopy and imaging. Biophysical Journal. 1994;66(1):259-267.

28. Roy M, Dumont M, Houde J, Morency F, Descoteaux M. Longitudinal assessment of neuroinflammation and axonal loss in white matter tracts in Alzheimer's disease. Alzheimer's \& Dementia. 2020;16(S5).

29. Braak H, Del Tredici K. Alzheimer's disease: intraneuronal alterations precede insoluble amyloid- $\beta$ formation. Neurobiology of Aging. 2004 Jul;25(6):713-8.

30. Wilson H, Politis M, Rabiner EA, Middleton LT. Novel PET Biomarkers to Disentangle Molecular Pathways across Age-Related Neurodegenerative Diseases. Cells. 2020 Dec 2;9(12):2581.

31. Liu Y, Nguyen M, Robert A, Meunier B. Metal Ions in Alzheimer's Disease: A Key Role or Not? Acc Chem Res. 2019 Jul 16;52(7):2026-35.

32. Innis R, Cunningham V, Delforge J, Fujita M, Gjedde A, Gunn R et al. Consensus Nomenclature for in vivo Imaging of Reversibly Binding Radioligands. Journal of Cerebral Blood Flow \& Metabolism. 2007;27(9):15331539.

33. Gunderson, L \& Tepper, J. Clinical Radiation Oncology 4th Edition. 2015.

34. for the Alzheimer's Disease Neuroimaging Initiative, for the ALFA Study, Salvadó G, Molinuevo JL, Brugulat-Serrat A, Falcon C, Grau-Rivera O, et al. Centiloid cut-off values for optimal agreement between PET and CSF core AD biomarkers. Alz Res Therapy. 2019 Dec;11(1):27.

35. Whittington A, Sharp D, Gunn R. Spatiotemporal Distribution of $\beta$-Amyloid in Alzheimer Disease Is the Result of Heterogeneous Regional Carrying Capacities. Journal of Nuclear Medicine. 2017;59(5):822-827.

36. Whittington A, Gunn R. Amyloid Load: A More Sensitive Biomarker for Amyloid Imaging. Journal of Nuclear Medicine. 2018;60(4):536-540.

37. Whittington A, Gunn R. TauIQ: A Canonical Image Based Algorithm to Quantify Tau PET Scans. Journal of Nuclear Medicine. 2021;62(9):1292-1300.

38. Chételat G, Arbizu J, Barthel H, Garibotto V, Law I, Morbelli S, et al. AmyloidPET and 18F-FDG-PET in the diagnostic investigation of Alzheimer's disease and other dementias. The Lancet Neurology. 2020 Nov;19(11):951-62.

39. Vogel JW, Young AL, Oxtoby NP, Smith R, Ossenkoppele R, Strandberg OT, et al. Characterizing the spatiotemporal variability of Alzheimer's disease pathology [Internet]. Neurology; 2020 Aug [cited 2021 Jul 11]. Available from: http: / / medrxiv.org/lookup/doi/10.1101/2020.08.20.20176883

40. Ossenkoppele R, Lyoo CH, Sudre CH, Westen D, Cho H, Ryu YH, et al. Distinct tau PET patterns in atrophy-defined subtypes of Alzheimer's disease. Alzheimer's \& Dementia. 2020 Feb;16(2):335-44.

41. La Joie R, Visani AV, Baker SL, Brown JA, Bourakova V, Cha J, et al. Prospective longitudinal atrophy in Alzheimer's disease correlates with the intensity and topography of baseline tau-PET. Sci Transl Med. 2020 Jan $1 ; 12(524)$.

42. Alzheimer's Disease Neuroimaging Initiative (ADNI), Franzmeier N, Neitzel J, Rubinski A, Smith R, Strandberg O, et al. Functional brain architecture is associated with the rate of tau accumulation in Alzheimer's disease. Nat Commun. 2020 Dec;11(1):347.

43. Krishnadas, N., Dore, V., Mulligan, R., Tyrrell, R., Bozinovski, S., Huang, K., Lamb, F., Burnham, S., Villemagne, V., \& Rowe, C. 18F-MK6240 longitudinal tau PET in ageing and Alzheimer's disease. 2021 Retrieved from: https: / /jnm. snmjournals.org/content/62/supplement_1/105

44. Mueller A, Bullich S, Barret O, Madonia J, Berndt M, Papin C, et al. Tau PET imaging with 18F-PI-2620 in Patients with Alzheimer Disease and Healthy Controls: A First-in-Humans Study. J Nucl Med. 2020 Jun;61(6):911-9.

45. Mattsson-Carlgren N, Andersson E, Janelidze S, Ossenkoppele R, Insel P, Strandberg $\mathrm{O}$, et al. $\mathrm{A} \beta$ deposition is associated with increases in soluble and phosphorylated tau that precede a positive Tau PET in Alzheimer's disease. Sci Adv. 2020 Apr;6(16):eaaz2387.

46. Rabin J, Yang H, Schultz A, Hanseeuw B, Hedden T, Viswanathan A et al. Vascular Risk and $\beta$-Amyloid Are Synergistically Associated with Cortical Tau. Annals of Neurology. 2019;85(2):272-279.

47. Cummings J, Aisen P, Lemere C, Atri A, Sabbagh M, Salloway S. Aducanumab produced a clinically meaningful benefit in association with amyloid lowering. Alzheimer's Research \& Therapy. 2021;13(1)

48. Pascoal T, Kang M, Therriault J, Benedet A, Chamoun M, Lussier F et al. Medial temporal neuroinflammation unleashes tau spreading over the neocortex. Alzheimer's \& Dementia. 2020;16(S4).

49. Doré V, Krishnadas N, Bourgeat P, Huang K, Li S, Burnham S et al. Relationship between amyloid and tau levels and its impact on tau spreading. European Journal of Nuclear Medicine and Molecular Imaging. 2021;

50. Eme-Scolan E, Dando SJ. Tools and Approaches for Studying Microglia In 
vivo. Front Immunol. 2020 Oct 7;11:583647.

51. Pomilio C, Gorojod RM, Riudavets M, Vinuesa A, Presa J, Gregosa A, et al. Microglial autophagy is impaired by prolonged exposure to $\beta$-amyloid peptides: evidence from experimental models and Alzheimer's disease patients. GeroScience. 2020 Apr;42(2):613-32.

52. Ismail R, Parbo P, Madsen LS, Hansen AK, Hansen KV, Schaldemose JL, et al. The relationships between neuroinflammation, beta-amyloid and tau deposition in Alzheimer's disease: a longitudinal PET study. J Neuroinflammation. 2020 Dec;17(1):151.

53. Calsolaro V, Matthews P, Donat C, Livingston N, Femminella G, Guedes S et al. Astrocyte reactivity with late-onset cognitive impairment assessed in vivo using 11C-BU99008 PET and its relationship with amyloid load. Molecular Psychiatry. 2021;.

54. Mansur, A., Rabiner, E., Comley, R., Lewis, Y., Middleton, L., Huiban, M., Passchier, J., Tsukada, H. and Gunn, R., 2019. Characterization of 3 PET Tracers for Quantification of Mitochondrial and Synaptic Function in Healthy Human Brain: 18F-BCPP-EF, 11C-SA-4503, and 11C-UCB-J. Journal of Nuclear Medicine, 61(1), pp.96-103.

55. Terada T, Obi T, Bunai T, Matsudaira T, Yoshikawa E, Ando I et al. In vivo mitochondrial and glycolytic impairments in patients with Alzheimer disease. Neurology. 2020;94(15):e1592-e1604.

56. O'Dell R, Mecca A, Chen M, Naganawa M, Toyonaga T, Lu Y et al. Association of $\mathrm{A} \beta$ deposition and regional synaptic density in early Alzheimer's disease: a PET imaging study with [11C]UCB-J. Alzheimer's Research \& Therapy. 2021;13(1).

57. Chen M, Mecca A, Naganawa M, Finnema S, Toyonaga T, Lin S et al. Assessing Synaptic Density in Alzheimer Disease With Synaptic Vesicle Glycoprotein 2A Positron Emission Tomographic Imaging. JAMA Neurology. 2018;75(10):1215.

58. Koronyo-Hamaoui M, Koronyo Y, Ljubimov A, Miller C, Ko M, Black K et al. Identification of amyloid plaques in retinas from Alzheimer's patients and noninvasive in vivo optical imaging of retinal plaques in a mouse model. NeuroImage. 2011;54:S204-S217.

59. Blanks J, Schmidt S, Torigoe Y, Porrello K, Hinton D, Blanks R. Retinal pathology in Alzheimer's disease. II. Regional neuron loss and glial changes in GCL. Neurobiology of Aging. 1996;17(3):385-395.

60. Liao H, Zhu Z, Peng Y. Potential Utility of Retinal Imaging for Alzheimer's Disease: A Review. Frontiers in Aging Neuroscience. 2018;10.

61. Chiasseu M, Alarcon-Martinez L, Belforte N, Quintero H, Dotigny F Destroismaisons $\mathrm{L}$ et al. Tau accumulation in the retina promotes early neuronal dysfunction and precedes brain pathology in a mouse model of Alzheimer's disease. Molecular Neurodegeneration. 2017;12(1).

62. Mahajan D, Votruba M. Can the retina be used to diagnose and plot the progression of Alzheimer's disease?. Acta Ophthalmologica. 2017;95(8):768777.
63. Chan V, Sun Z, Tang S, Chen L, Wong A, Tham C et al. Spectral-Domain OCT Measurements in Alzheimer's Disease. Ophthalmology. 2019;126(4):497-510.

64. Ko F, Muthy Z, Gallacher I, Sudlow C, Rees G, Yang Q et al. Association of Retinal Nerve Fiber Layer Thinning With Current and Future Cognitive Decline. JAMA Neurology. 2018;75(10):1198.

65. Mutlu U, Colijn J, Ikram M, Bonnemaijer P, Licher S, Wolters F et al Association of Retinal Neurodegeneration on Optical Coherence Tomography With Dementia. JAMA Neurology. 2018;75(10):1256.

66. Mukherjee C, Al-Fahad Q, Elsherbiny S. The role of optical coherence tomography in therapeutics and conditions, which primarily have systemic manifestations: a narrative review. Therapeutic Advances in Ophthalmology. 2019;11:251584141983115.

67. Dumitrascu O, Lyden P, Torbati T, Sheyn J, Sherzai A, Sherzai D et al. Sectoral segmentation of retinal amyloid imaging in subjects with cognitive decline. Alzheimer's \& Dementia: Diagnosis, Assessment \& Disease Monitoring. 2020;12(1).

68. Ngolab J, Donohue M, Belsha A, Salazar J, Cohen P, Jaiswal S et al. Feasibility study for detection of retinal amyloid in clinical trials: The Anti-Amyloid Treatment in Asymptomatic Alzheimer's Disease (A4) trial. Alzheimer's \& Dementia: Diagnosis, Assessment \& Disease Monitoring. 2021;13(1).

69. Snyder P, Johnson L, Lim Y, Santos C, Alber J, Maruff P et al. Nonvascular retinal imaging markers of preclinical Alzheimer's disease. Alzheimer's \& Dementia: Diagnosis, Assessment \& Disease Monitoring. 2016;4(1):169-178

70. Udeh-Momoh C, Watermeyer T, Price G, de Jager Loots C, ReglinskaMatveyev N, Ropacki M et al. Protocol of the Cognitive Health in Ageing Register: Investigational, Observational and Trial Studies in Dementia Research (CHARIOT): Prospective Readiness cOhort (PRO) SubStudy. BMJ Open. 2021;11(6):e043114.

71. Pan D, Zeng A, Jia L, Huang Y, Frizzell T, Song X. Early Detection of Alzheimer's Disease Using Magnetic Resonance Imaging: A Novel Approach Combining Convolutional Neural Networks and Ensemble Learning. Front Neurosci. 2020 May 13;14:259.

72. Venugopalan J, Tong L, Hassanzadeh HR, Wang MD. Multimodal deep learning models for early detection of Alzheimer's disease stage. Sci Rep. 2021 Dec;11(1):3254.

73. Knudsen G, Ganz M, Appelhoff S, Boellaard R, Bormans G, Carson R et al Guidelines for the content and format of PET brain data in publications and archives: A consensus paper. Journal of Cerebral Blood Flow \& Metabolism. 2020;40(8):1576-1585.

How to cite this article: J. Ford, D. Kafetsouli, H. Wilson, et al. At a Glance: An Update on Neuroimaging and Retinal Imaging in Alzheimer's Disease and Related Research. J Prev Alz Dis 2022;1(9):67-76, http:/ /dx.doi.org/10.14283/ jpad.2022.7 